\title{
Efecto de la fase fenológica de verano sobre algunas características del ensamble de Diptera registrado en una vega altoandina del desierto transicional de Chile
}

\author{
Effect of the summer phenological phase on some characteristics of the assemblage \\ of Diptera registered in an Andean wet pasture of the transitional desert of Chile
}

\author{
J. Cepeda-Pizarro ${ }^{1 *}$, M. Pola P. ${ }^{2}$, C.R. González ${ }^{3}$
}

\begin{abstract}
RESUMEN
Uno de los grupos taxonómicos dominantes de invertebrados a encontrar en los prados húmedos altoandinos del desierto transicional de Chile lo constituyen los dípteros. En humedales, estos insectos desempeñan roles importantes como agentes polinizadores, descomponedores y recursos tróficos para vertebrados acuáticos. Adicionalmente, a Diptera se le reconoce la potencialidad de indicador de la condición de "salud" de los ambientes terrestres húmedos, atributo importante para el caso de las vegas altoandinas del norte de Chile, debido al estrés hídrico a que están actualmente sujetas. Se estudió, mediante trampas Barber y Malaise, el ensamble de Diptera asociado a la estación de crecimiento biológico de una vega altoandina localizada en el norte-centro de Chile. Los objetivos fueron a) documentar la estructura taxonómica del ensamble en términos de la composición de familias y de la abundancia relativa de sus miembros, $\mathrm{y}$ b) evaluar el efecto de la fase fenológica de verano sobre la riqueza- $\mathrm{F}$ (familias) del ensamble, sus relaciones de abundancia relativa y los indicadores de diversidad y equitabilidad-F. Las diferencias observadas en las relaciones de abundancia relativa fueron evaluadas con las pruebas PERMANOVA y t-pareada. La diversidad-F se estimó con los índices Simpsons D, Brillouin D, dominancia de Berger-Parker y Fisher. La equitabilidad-F se estimó con los índices Simpson E, Camargo, Smith-Wilson B y NHC. La similaridad entre ensambles se evaluó con la prueba ANOSIM. La contribución porcentual de las familias a las diferencias observadas se evaluó con la prueba SIMPER. Todos los análisis se realizaron con los datos $(\mathrm{x}+1)$ logarítmicamente transformados $(\log 10)$. Se capturaron 44.524 especímenes, con registro de 27 familias. Las pruebas aplicadas y los índices usados sustentan la idea que el verano medio fue la fase fenológica ambientalmente más favorable a la dipterofauna de la vega. Aparentemente, este efecto estaría relacionado más con el patrón térmico del área que con la disponibilidad de microhábitats.

Palabras clave: Andes semiáridos, insectos de montaña, pastizales húmedos, bioindicadores, factores ambientales.
\end{abstract}

\begin{abstract}
Diptera are one of the dominant taxonomic groups of animal invertebrates to be found in the highland wet pastures of the transitional desert of Chile. In wetlands, these insects play important roles as pollinators, decomposers and trophic resources for aquatic vertebrates. Additionally, Diptera are recognized to have the potentiality to be used as indicators of the health condition of wet terrestrial environments, important attribute in the case of Andean highland wet pastures given the water stress under which they are presently subjected. By using Barber and Malaise traps, the Diptera-assemblage associated to a highland wet pasture located in a transitional desert basin was investigated. This study was conducted during the growing season for the area. Its aims were to a) document the taxonomic structure of the assemblage in terms of the composition of Diptera-families and the relative abundance of its members, and $b$ ) evaluate the effect of the summer phenological phase on F-richness, relative abundance relationships, $F$-diversity and evenness. The PERMANOVA and t-pairwise tests were used to evaluate the effect of the summer phenological phase on the numerical relationships among families. The Simpsons D, Brillouin D, Berger-Parker dominance, and Fisher indices were used to estimate the F-diversity; in turn, the Simpson E, Camargo, Smith-Wilson B, and NHC indices estimated the F-evenness. Similarity between assemblages was compared by ANOSIM. The percent contribution by family to the observed differences between assemblages was computed by the SIMPER-routine. All analyses were done on the log-transformed data $(x+1 ; \log 10) .44524$ Diptera-representatives were collected throughout the study, with a record of 27 families. The tests and the indices support the idea that the middle-summer phase provided the best environmental conditions for the diptero-fauna of the wet pasture. Apparently, this effect would be more related to the thermal pattern of the area than to the micro-habitat availability.
\end{abstract}

Key words: Semi-arid Andes, highland insects, wet pastures, bio-indicators, environmental factors.

\footnotetext{
1 Departamento de Biología. Facultad de Ciencias, Universidad de La Serena. Benavente 980. La Serena, Chile.

2 Departamento de Biología. Universidad Autónoma de Madrid. C/Darwin, 228049 Madrid, España.

3 Instituto de Entomología. Universidad Metropolitana de Ciencias de la Educación. Santiago, Chile.

* Autor para correspondencia: jcepeda@userena.cl
} 


\section{Introducción}

Las vegas, un tipo de prado húmedo, son elementos comunes del paisaje altoandino del desierto transicional de Chile, donde conforman focos de biodiversidad para un conjunto diverso de organismos (Squeo et al., 2006a). Para el caso de los animales invertebrados que viven en ambientes de altamontaña, su presencia y éxito colonizador depende estrechamente de su capacidad de adaptación a las condiciones ambientales imperantes, generalmente extremas (Hinden et al., 2005). Uno de los grupos taxonómicos dominantes del ensamble de los animales invertebrados de los prados húmedos altoandinos del desierto transicional del Chile lo constituyen los dípteros (Cepeda-Pizarro \& Pola, 2013). En humedales, estos insectos desempeñan roles importantes como agentes polinizadores, descomponedores y recursos tróficos para la avifauna y otros vertebrados (Keiper et al., 2002). Adicionalmente, en atención a que muchas de sus especies requieren condiciones específicas para completar su desarrollo (Batzer \& Wissinger, 1996), a Diptera se le reconoce la potencialidad de indicador de la condición de "salud" de los ambientes terrestres húmedos (Rosenberg, 1992), atributo importante, debido al estrés hídrico al que están actualmente sujetas las vegas altoandinas del norte de Chile (Contreras, 2002). Los objetivos del trabajo fueron: a) documentar la estructura taxonómica del ensamble en términos de la composición de familias y de la abundancia relativa de sus miembros, y b) evaluar el efecto de la fase fenológica de verano sobre la riqueza de familias del ensamble (riqueza F), sus relaciones de abundancia relativa y los indicadores de diversidad y equitabilidad F. Se estableció como hipótesis de trabajo que la fase fenológica del humedal, en su período de máxima productividad biológica (i. e., verano), influye en las características del ensamble.

\section{Materiales y Métodos}

\section{Vega estudiada}

El estudio se realizó en la vega Tambo-Puquíos (VTP), ubicada en la microcuenca del río Vacas Heladas, en la alta montaña de la hoya hidrográfica del río Elqui (Figura 1). VTP tiene una superficie de $10 \mathrm{~km}^{2}$, con una extensión de $\sim 6 \mathrm{~km}$, posee una gradiente de elevación de $\sim 200 \mathrm{~m}$, desde 3.800 a
$4.000 \mathrm{msnm}$. Paisajísticamente, VTP está conformada por un manto de vegetación cespitosa dominada por hierbas perennes como Carex maritima, Puccinella oresigena y Deschampsia caespitosa, el que incluye sitios inundados y pequeñas pozas (Figura 2). Pegado al borde de la vega corre un cuerpo permanente de agua. Para este estudio se seleccionaron tres sitios de monitoreo. Los criterios para su selección fueron estructura similar en términos de cobertura vegetal y condiciones de humedad (p.e., presencia de pozas y lugares inundados). Estos sitios fueron Puquíos (SPQ en adelante, $3.830 \mathrm{msnm}$ ), sitio Manguera (SMG, $3.950 \mathrm{msnm}$ ) y sitio Tambo (STB, 3.980 $\mathrm{msnm})$. En el centro de cada uno de ellos se instaló

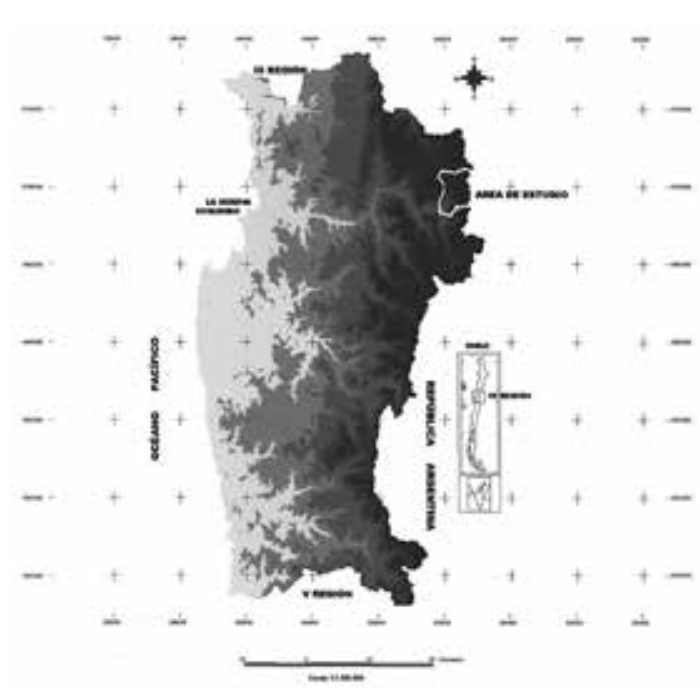

Figura 1. Área de estudio en la hoya hidrográfica del río Elqui (Región de Coquimbo, Chile) (3.800-4.000 msnm).

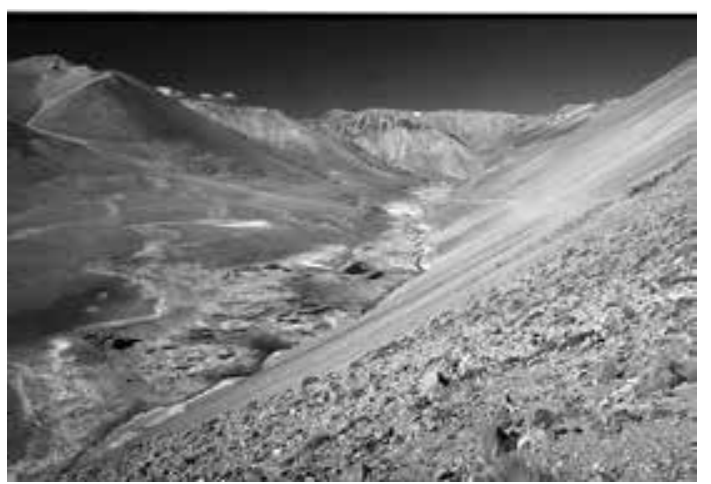

Figura 2. Vista general de la sección inferior $(3.830 \mathrm{msnm}) \mathrm{de}$ la vega Tambo-Puquíos. Los parches más oscuros corresponden a pozas superficiales; las manchas blancas incluidas en la vega, a depósitos de sulfato. 
una parcela de monitoreo de $5 \times 4 \mathrm{~m}$, dentro de esta se instaló una cuadrícula de 20 trampas Barber. Aledaña a ella se emplazó una trampa Malaise, según lo descrito en Cepeda-Pizarro \& Pola (2013).

\section{Metodología de muestreo y procesamiento de la información}

En atención a antecedentes referidos a la actividad biológica de la vega (Squeo et al., 2006b), las campañas de captura se realizaron durante el período de verano. En consideración a las características de precipitación y temperatura del aire de la microcuenca de localización, estas campañas cubrieron las fases fenológicas de verano inicial (desde el 21 de diciembre al 21 de enero); verano medio (desde el 21 de enero al 21 de febrero) y verano final (desde el 21 de febrero al 21 de marzo). Las trampas permanecieron continuamente activas por 15 días en cada una de las fases fenológicas. Durante el receso, las trampas Barber fueron inactivadas y las trampas Malaise fueron retiradas para su reacondicionamiento. Cada vez y por tipo de trampa, el líquido letal conteniendo los especímenes capturados fue retirado y depositado en bolsas plásticas ad hoc. Estas fueron trasladadas al laboratorio en contenedores refrigerados y mantenidos en esa condición hasta su procesamiento. Las comparaciones realizadas se basaron en las sumas de los aportes de cada tipo de trampa. En la identificación taxonómica del material capturado se usó la colección de referencia depositada en el Instituto de Entomología de la Universidad Metropolitana. Siguiendo a Timms et al. (2014) y al grado del conocimiento taxonómico actualmente disponible para este taxón en Chile, la categoría usada como referencia fue familia, según McAlpine et al. (1981, 1987). El diseño usado en el estudio fue seudoexperimental, con fase fenológica como la variable seudoexperimental (fase fenológica $=3$ ) y sitio como réplica $($ sitio $=3$ ) (Steel \& Torrie, 1989). La evaluación del efecto de la fase fenológica sobre las relaciones de abundancia relativa entre familias se realizó con la prueba PERMANOVA y t-pareada (Anderson, 2005). El análisis PERMANOVA se basó en el índice Bray-Curtis de disimilitud (disimilaridad), con los datos originales $(x+1)$ transformados logarítmicamente (log 10$)$. La riqueza $\mathrm{F}$ (i. e., familias) fue estimada según los estimadores Chao \& Lee 1 (ACE), Jackknife 1 y Rarefacción (agrupada, finita). La diversidad del ensamble de familias asociadas a la vega (diversidad-F) fue estimada según los siguientes índices: Simpson (D), Brillouin (D), dominancia (Berger-Parker) y alfa (Fisher). La equitabilidad del ensamble (equitabilidad-F) fue estimada mediante Simpson (E), Camargo, Smith \& Wilson (B) y NHC. Los cálculos fueron realizados con el programa Species Diversity and Richness IV (Seaby \& Henderson, 2006). Complementariamente, los resultados se compararon mediante las pruebas ANOSIM y SIMPER sobre los datos crudos logarítmicamente transformados $(\log x+1)$. Para este último propósito los cálculos fueron realizados con el programa CAP (Henderson \& Seaby, 2008).

\section{Resultados y Discusión}

\section{El ensamble de familias de Diptera}

En total se capturaron 44.524 especímenes, con registro de 27 familias (Tabla 1). Dieciocho de ellas mostró abundancia relativa porcentual total $\geq 0,1 \%$. El 94,3\% de las capturas fue aportada por 6 familias; dos de ellas (Chironomidae y Muscidae) contribuyeron con el $64 \%$. Nueve familias mostraron abundancia relativa $<0,1 \%$. Debido a este nivel de abundancia y en el contexto de este trabajo, estas familias se consideraron escasas o raras en el sitio de estudio. Entre fases fenológicas los aportes porcentuales totales fueron $59,7 \%$ (verano inicial), $27,6 \%$ (verano medio) y $12,6 \%$ (verano final) (Tabla 1). Mientras el número de familias exclusivas por fase fenológica fluctuó entre 4-5 familias; el número de familias comunes fluctuó entre 19-20 familias, con tendencia a aumentar en el curso del verano (Tablas 2 a 4). El número predicho para el total de familias a encontrar en la vega Tambo-Puquíos varió entre 27 (Chao \& Lee 1 ; rarefacción) y 30 (jackknifing) (Tabla 5). Mientras el número total observado de familias por fase fenológica de verano disminuyó en el sentido del curso del verano, desde 22 (verano inicial) a 20 (verano final), el número de familias con abundancia relativa $\geq 0,1 \%$ mostró un máximo de 13 familias en la fase verano medio (Tabla 1). Respecto de la riqueza-F estimada por fase fenológica de verano, mientras la tendencia mostrada por la rarefacción fue consistente con el F-observado, ACE y jackknifing lo fueron con las familias $\geq 0,1 \%$ (Tabla 5). 
Tabla 1. Contribución de familias de Diptera registradas por fase fenológica de verano de la vega altoandina Tambo-Puquíos del desierto transicional de Chile.

\begin{tabular}{|c|c|c|c|c|c|c|c|c|}
\hline \multirow{3}{*}{ Familia } & \multicolumn{6}{|c|}{ Fase fenológica* } & \multirow{2}{*}{\multicolumn{2}{|c|}{ Total }} \\
\hline & \multicolumn{2}{|c|}{ VI } & \multicolumn{2}{|c|}{$\mathrm{VM}$} & \multicolumn{2}{|c|}{ VF } & & \\
\hline & $\mathrm{n}$ & $\%$ & $\mathrm{n}$ & $\%$ & $\mathrm{n}$ & $\%$ & $\mathrm{n}$ & $\%$ \\
\hline Agromyzidae & 6 & $<0,1$ & 0 & 0 & 59 & 0,1 & 65 & 0,1 \\
\hline Anthomyiidae & 7 & $<0,1$ & 70 & 0,2 & 0 & 0 & 77 & 0,2 \\
\hline Anthomyzidae & 0 & 0,0 & 45 & 0,1 & 15 & $<0,1$ & 60 & 0,1 \\
\hline Bombyliidae & 0 & 0,0 & 4 & $<0,1$ & 0 & 0 & 4 & $<0,1$ \\
\hline Calliphoridae & 8 & $<0,1$ & 16 & $<0,1$ & 2 & 0 & 26 & 0,1 \\
\hline Cecidomyiidae & 12 & $<0,1$ & 35 & 0,1 & 18 & $<0,1$ & 65 & 0,2 \\
\hline Ceratopogonidae & 310 & 0,7 & 374 & 0,8 & 224 & 0,5 & 908 & 2,0 \\
\hline Chironomidae & 9450 & 21,2 & 2614 & 5,9 & 1384 & 3,1 & 13448 & 30,2 \\
\hline Chloropidae & 383 & 0,9 & 262 & 0,6 & 1 & $<0,1$ & 646 & 1,5 \\
\hline Dolichopodidae & 845 & 1,9 & 1205 & 2,7 & 4 & $<0,1$ & 2054 & 4,6 \\
\hline Empididae & 25 & 0,1 & 181 & 0,4 & 1 & $<0,1$ & 207 & 0,5 \\
\hline Ephydridae & 1274 & 2,9 & 369 & 0,8 & 545 & 1,2 & 2188 & 4,9 \\
\hline Heleomyzidae & 32 & 0,1 & 1 & $<0,1$ & 3 & $<0,1$ & 36 & 0,1 \\
\hline Muscidae & 10422 & 23,4 & 4154 & 9,3 & 599 & 1,4 & 15175 & 34,1 \\
\hline Mycetophilidae & 0 & 0,0 & 0 & 0 & 7 & $<0,1$ & 7 & $<0,1$ \\
\hline Phoridae & 54 & 0,1 & 93 & 0,2 & 18 & $<0,1$ & 165 & 0,4 \\
\hline Piophilidae & 17 & $<0,1$ & 0 & 0 & 0 & 0 & 17 & $<0,1$ \\
\hline Sarcophagidae & 2 & $<0,1$ & 0 & 0 & 0 & 0 & 2 & $<0,1$ \\
\hline Sciaridae & 60 & 0,1 & 42 & $<0,1$ & 84 & 0,2 & 186 & 0,4 \\
\hline Simuliidae & 1 & $<0,1$ & 0 & 0 & 0 & 0 & 1 & $<0,1$ \\
\hline Sphaeroceridae & 1200 & 2,7 & 114 & 0,3 & 13 & $<0,1$ & 1327 & 3,0 \\
\hline Stratiomyidae & 0 & 0,0 & 1 & 0 & 0 & 0 & 1 & $<0,1$ \\
\hline Syrphidae & 2476 & 5,6 & 2688 & 6 & 2481 & 5,6 & 7645 & 17,2 \\
\hline Tabanidae & 0 & 0,0 & 22 & $<0,1$ & 0 & 0 & 22 & $<0,1$ \\
\hline Tachinidae & 2 & $<0,1$ & 12 & $<0,1$ & 152 & 0,3 & 166 & 0,4 \\
\hline Therevidae & 2 & $<0,1$ & 0 & 0 & 6 & $<0,1$ & 8 & $<0,1$ \\
\hline Tipulidae & 11 & $<0,1$ & 1 & $<0,1$ & 6 & $<0,1$ & 18 & $<0,1$ \\
\hline Total N & 26599 & 59,7 & 12303 & 27,6 & 5622 & 12,6 & 44524 & 100 \\
\hline Total-F & & 22,0 & & 21,0 & & 20,0 & & 27 \\
\hline Total-F $(\geq 0,1 \%)$ & & 12 & & 13 & & 8 & & 18 \\
\hline
\end{tabular}

*VI: verano inicial (21.12 al 21.01); VM: verano medio (21.01 al 21.02); VF: verano final (21.02 al 21.03).

Tabla 2. Distribución de familias de Diptera entre fases fenológicas de verano de la vega Tambo-Puquíos. Comparación: verano inicial/verano medio.

\begin{tabular}{|c|c|c|}
\hline \multicolumn{3}{|c|}{ VI/VM } \\
\hline Únicas a VI & Comunes & Únicas a VM \\
\hline 5 & 18 & 4 \\
\hline $\begin{array}{l}\text { Agromyzidae } \\
\text { Piophilidae } \\
\text { Sarcophagidae } \\
\text { Simuliidae } \\
\text { Therevidae }\end{array}$ & $\begin{array}{l}\text { Anthomyiidae } \\
\text { Calliphoridae } \\
\text { Cecidomyiidae } \\
\text { Ceratopogonidae } \\
\text { Chironomidae } \\
\text { Chloropidae } \\
\text { Dolichopodidae } \\
\text { Empididae } \\
\text { Ephydridae } \\
\text { Heleomyzidae } \\
\text { Muscidae } \\
\text { Mycetophilidae } \\
\text { Phoridae } \\
\text { Sciaridae } \\
\text { Sphaeroceridae } \\
\text { Syrphidae } \\
\text { Tachinidae } \\
\text { Tipulidae }\end{array}$ & $\begin{array}{l}\text { Anthomyzidae } \\
\text { Bombyliidae } \\
\text { Stratiomyidae } \\
\text { Tabanidae }\end{array}$ \\
\hline
\end{tabular}

Tabla 3. Distribución de familias de Diptera entre fases fenológicas de verano de la vega Tambo-Puquíos. Comparación verano inicial/verano final.

\begin{tabular}{|c|c|c|}
\hline \multicolumn{3}{|c|}{$\mathrm{VI} / \mathrm{VF}$} \\
\hline Únicas a VI & Comunes & Únicas a VF \\
\hline 4 & 19 & 4 \\
\hline $\begin{array}{l}\text { Anthomyiidae } \\
\text { Piophilidae } \\
\text { Sarcophagidae } \\
\text { Simuliidae }\end{array}$ & $\begin{array}{l}\text { Agromyzidae } \\
\text { Calliphoridae } \\
\text { Cecidomyiidae } \\
\text { Ceratopogonidae } \\
\text { Chironomidae } \\
\text { Chloropidae } \\
\text { Dolichopodidae } \\
\text { Empididae } \\
\text { Ephydridae } \\
\text { Heleomyzidae } \\
\text { Muscidae } \\
\text { Mycetophilidae } \\
\text { Phoridae } \\
\text { Sciaridae } \\
\text { Sphaeroceridae } \\
\text { Syrphidae } \\
\text { Tachinidae } \\
\text { Therevidae } \\
\text { Tipulidae }\end{array}$ & $\begin{array}{l}\text { Anthomyzidae } \\
\text { Bombyliidae } \\
\text { Stratiomyidae } \\
\text { Tabanidae }\end{array}$ \\
\hline
\end{tabular}


Tabla 4. Distribución de familias de Diptera entre fases fenológicas de verano de la vega Tambo-Puquíos. Comparación verano medio/verano final.

\begin{tabular}{|c|c|c|}
\hline \multicolumn{3}{|c|}{$\mathrm{VM} / \mathrm{VF}$} \\
\hline Únicas a VM & Comunes & Únicas a VF \\
\hline 4 & 20 & 3 \\
\hline $\begin{array}{l}\text { Anthomyiidae } \\
\text { Bombyliidae } \\
\text { Stratiomyidae } \\
\text { Tabanidae }\end{array}$ & $\begin{array}{l}\text { Anthomyzidae } \\
\text { Calliphoridae } \\
\text { Cecidomyiidae } \\
\text { Ceratopogonidae } \\
\text { Chironomidae } \\
\text { Chloropidae } \\
\text { Dolichopodidae } \\
\text { Empididae } \\
\text { Ephydridae } \\
\text { Heleomyzidae } \\
\text { Muscidae } \\
\text { Phoridae } \\
\text { Piophilidae } \\
\text { Sarcophagidae } \\
\text { Sciaridae } \\
\text { Simuliidae }\end{array}$ & $\begin{array}{l}\text { Agromyzidae } \\
\text { Mycetophilidae } \\
\text { Therevidae }\end{array}$ \\
\hline
\end{tabular}

\section{Efecto de la fase fenológica de verano sobre la relaciones de abundancia entre familias $y$ algunas características del ensamble}

La prueba PERMANOVA mostró un efecto de la fase fenológica sobre las relaciones de abundancia entre familias ( $\left.\mathrm{p}_{\text {perm; 2,6 g.l. }}=0,016 ; \mathrm{p}_{\mathrm{MC} ; 2,6 \mathrm{~g} . \mathrm{l}}=0,034\right)$, particularmente en la comparación pareada verano inicial/verano final y un aparente efecto en la comparación verano medio/verano final (Tablas 6 y 7). El índice de disimilaridad B-C tomó valores entre $33,7 \%$ y $45,4 \%$ en la comparación entre ensambles (Tabla 7). En términos de la diversidad estimada F (Figura 3), mientras los índices Simpsons D y Brillouin mostraron valores más altos en la fase verano medio, el índice B-P de dominancia mostró la tendencia opuesta. A su vez, el índice $\alpha$ de Fisher mostró una tendencia ascendente aparentemente asintótica. La comparación mediante boostraping de la diversidad estimada F mostró que varias de las

Tabla 5. Riqueza estimada de familias (F) del ensamble de Diptera registrado en la vega Tambo-Puquíos en sus fases fenológicas de verano.

\begin{tabular}{lllll}
\hline \multirow{2}{*}{ Estimador } & \multicolumn{3}{c}{ Fase fenológica } & Total F \\
\cline { 2 - 4 } & \multicolumn{1}{c}{ VI } & \multicolumn{1}{c}{ VM } & VF & 27 \\
\hline Chao \& Lee 1 (ACE) & 18,75 & 19,01 & 16,87 & 29,67 \\
Jackknifing 1* & $21,67(5,48)$ & $23,41(5,64)$ & $18,48(4,06)$ & $27(0,007)$ \\
Rarefacción (agrupada, finita)** & $21,41(0,683)$ & $20,4(0,670)$ & $19,25(0,742)$ & 27 \\
$\mathrm{~N}^{\circ}$ F observadas & 22 & 21 & 20 & 18 \\
$\mathrm{~N}^{\circ} \mathrm{F} \geq 0,1 \%$ & 12 & 13 & 8 & \\
\hline
\end{tabular}

Entre paréntesis: *(de);**(ee).

Tabla 6. Evaluación del efecto de la fase fenológica de verano sobre las relaciones de abundancia relativa de familias de Diptera registradas en la vega Tambo-Puquíos.

\begin{tabular}{lcccccc}
\hline FV* & GL & SC & MC & F & P (perm) & P (MC) \\
\hline Fase & 2 & 3092,8 & 1546,4 & 3,0252 & 0,016 & 0,034 \\
Residual & 6 & 3067,1 & 5112 & & & \\
Total & 8 & 6160 & & & \\
\hline
\end{tabular}

*Prueba PERMANOVA; entero usado como semilla: 5; número de permutaciones: 999.

Tabla 7. Valores de la prueba de t-pareada y del índice de disimilaridad B-C para comparaciones pareadas de ensambles según sus fases fenológicas de verano.

\begin{tabular}{lccll}
\hline Comparación & $\mathrm{t}$ & $\mathrm{P}$-perm & \multicolumn{1}{c}{ P-MC } & $\mathrm{B}-\mathrm{C}$ \\
\hline VI/VM & 1,14 & 0,2780 & 0,3220 & 33,7 \\
VI/VF & 2,31 & 0,0990 & $0,029(1)$ & 45,4 \\
VM/VF & 1,71 & 0,0890 & $0,098(2)$ & 40,2 \\
\hline
\end{tabular}

(1) significativo al $5 \%$; (2) cercano al valor crítico $(0,05 \%)$. diferencias observadas fueron significativas (Tabla 8). En términos de las características de equitabilidad del ensamble, mientras el índice Simpson E mostró un valor mayor en la fase verano medio $(0,217)$ respecto del verano inicial $(0,155)$ y verano final $(0,179)$, los índices restantes mostraron curvas ascendentes, aparentemente asintóticas (Figura 4). Como observado con la diversidad, la comparación mediante boostraping de la equitabilidad mostró 


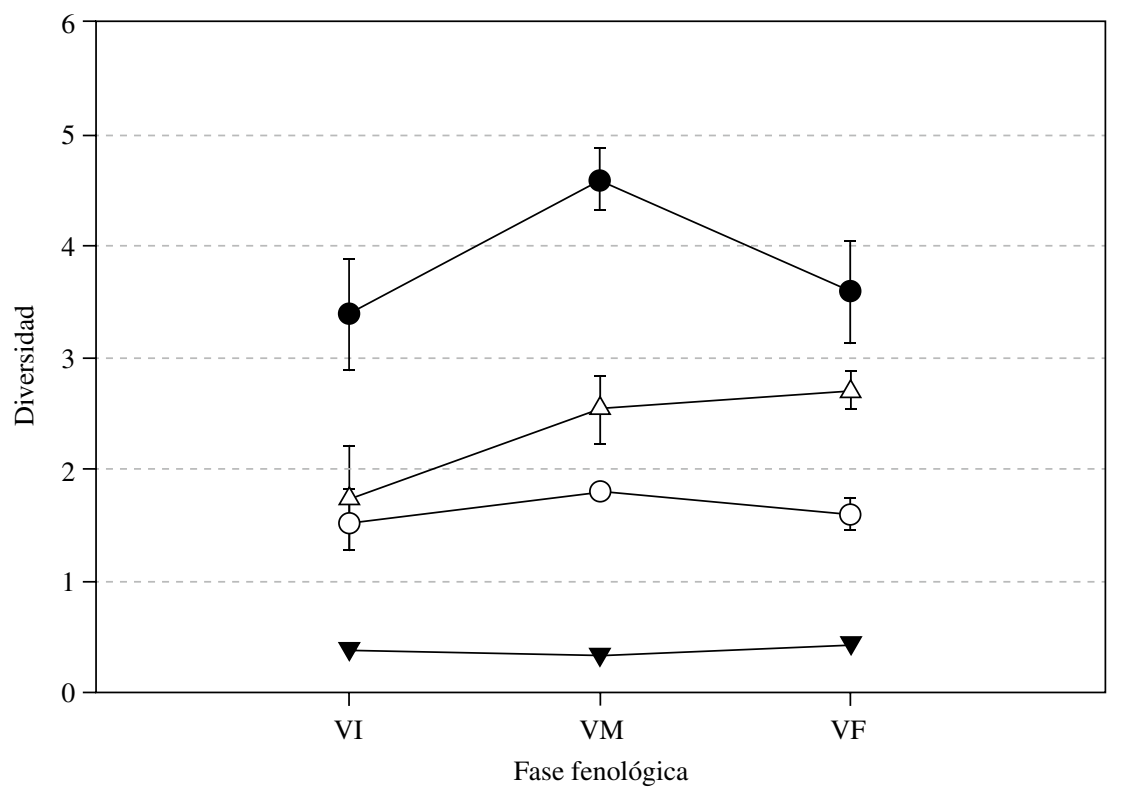

- Simpsons D $-\mathrm{O}-$ Brillouin D $\rightarrow$ Dominancia B-P $\triangle$ Fisher B-P

Figura 3. Variación de la estimación de la diversidad-F de un ensamble de familias de Diptera registrado en la vega Tambo-Puquíos durante el verano, según los índices Simpsons D; Brillouin D, dominancia de Berger-Parker y $\alpha$ Fisher. La barra vertical corresponde al error estándar de la estimación. VI: verano inicial; VM: verano medio; VF: verano final.

Tabla 8. Comparación entre fases fenológicas de verano de la diversidad y la equitabilidad del ensamble de Diptera registrado en la vega Tambo-Puquíos.

\begin{tabular}{|c|c|c|c|c|c|}
\hline \multirow{2}{*}{ Parámetro } & \multirow{2}{*}{ Índice } & \multirow{2}{*}{ Fase fenológica } & \multicolumn{3}{|c|}{ Fase fenológica } \\
\hline & & & VI & VM & VF \\
\hline \multirow{8}{*}{ Diversidad } & \multirow{3}{*}{ Simpsons D } & VI & & & \multirow{3}{*}{ DS:VM>VF } \\
\hline & & VM & DS:VM>VI & & \\
\hline & & VF & DS:VF>VI & & \\
\hline & \multirow{3}{*}{ Brillouin } & VI & & & \multirow{3}{*}{ DS:VM>VF } \\
\hline & & VM & DS:VM>VI & & \\
\hline & & VF & DS:VF>VI & & \\
\hline & \multirow[b]{2}{*}{ B-P dominancia } & VI & & \multicolumn{2}{|c|}{ DS:VI>VM } \\
\hline & & $\begin{array}{l}\text { VM } \\
\text { VF }\end{array}$ & DS:VF>VI & DS:VF $>V M$ & \\
\hline \multirow{12}{*}{ Equitabilidad } & & VI & & & \\
\hline & Simpson E & VM & DS:VM>VI & & DS:VM>VF \\
\hline & & VF & NS & & \\
\hline & \multirow{3}{*}{ Camargo } & VF & & \multirow[b]{3}{*}{ DS:VF>VM } & \\
\hline & & VM & DS:VM>VI & & \\
\hline & & VF & DS:VF>VI & & \\
\hline & \multirow{3}{*}{ S \& W B } & VI & & \multirow[b]{3}{*}{ NS } & \\
\hline & & VM & NS & & \\
\hline & & VF & DS:VF>VI & & \\
\hline & \multirow{3}{*}{$\mathrm{NHC}$} & VI & & \multirow{3}{*}{ DS:VF>VM } & \\
\hline & & VM & DS:VM>VI & & \\
\hline & & VF & DS:VF>VI & & \\
\hline
\end{tabular}

*DS: diferencia significativa; NS: no significativa; ambas al 5\%. 


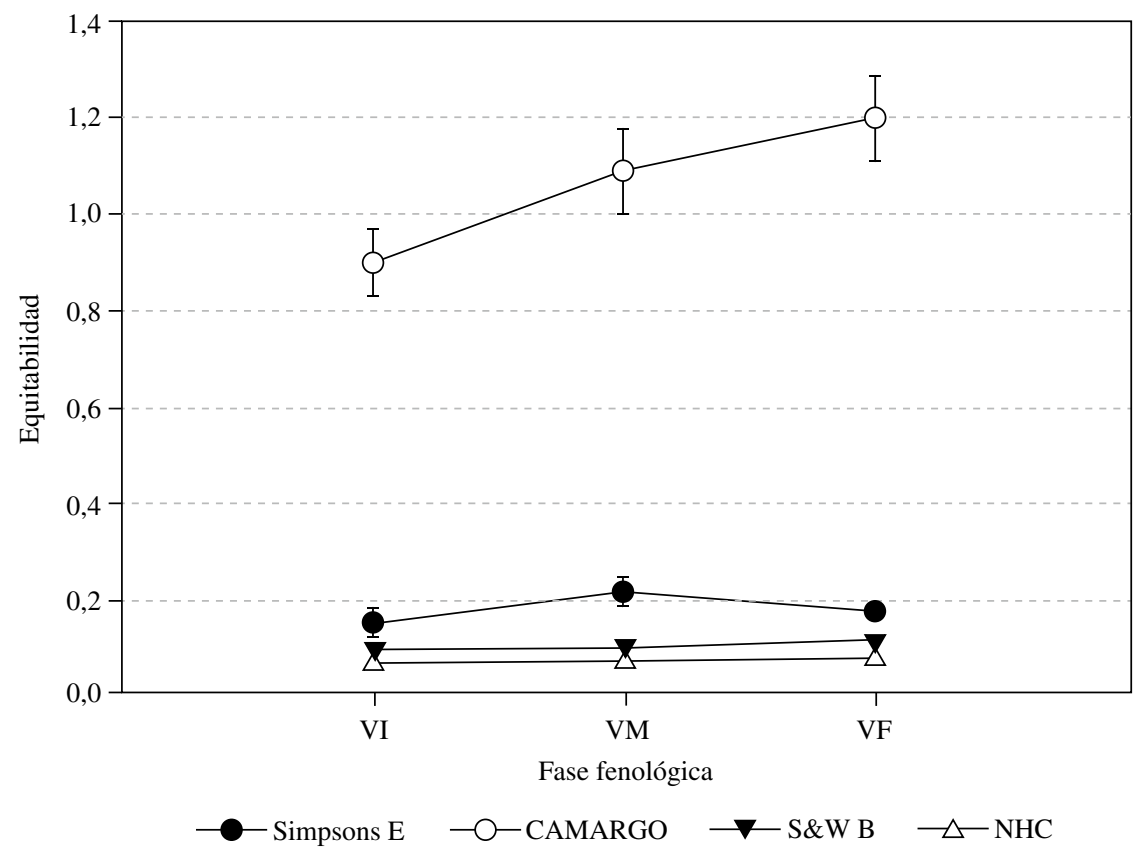

Figura 4. Variación de la estimación de la equitabilidad-F de un ensamble de familias de Diptera registrado en la vega TamboPuquíos durante el verano, según los índices Simpsons E, Camargo; Smith \& Wilson's B y NHC. La barra vertical corresponde al error estándar de la estimación. Nomenclatura para VI, VM y VF como en la Figura 3.

que varias de las diferencias observadas fueron significativas (Tabla 8). El promedio global de disimilitud por fase fenológica, estimado mediante el análisis SIMPER, tendió a un valor más alto en la fase fenológica de verano medio $(0,53)$, comparado con aquel que mostraron las fases verano inicial $(0,43)$ y verano final $(0,34)$. Se observó una cierta heterogeneidad entre sitios por fase fenológica $($ sitio=3). Ella pudo haberse debido a efectos derivados de las diferencias en altitud (p. e, $200 \mathrm{~m}$ entre el sitio de posición altitudinal más baja y aquel de posición más alta) o a diferencias estructurales no observadas al momento de su selección como sitio de observación (p. e., diferencias en la distribución del mosaico de parches húmedos versus parches secos). El porcentaje global de disimilitud de la comparación entre fases fenológicas fue relativamente bajo, fluctuando entre 47,41 y 63,73\% (Tabla 9). No obstante, las comparaciones verano inicial/ verano final y verano medio/verano final resultaron significativas con $\mathrm{p}<0,05 \%$; no así la comparación verano inicial/verano medio ( $\mathrm{p}<0,35 \%$ ) (ANOSIM, R-global: 0,$679 ; \mathrm{p} \leq 0,01 ;$ nivel $0,1 \%)$. En la Tabla 9 se muestra el comportamiento por fase fenológica de la abundancia promedio de las familias con mayor contribución porcentual a la explicación de las diferencias entre ensambles. En general, cuatro familias fueron los elementos comunes que aportaron el mayor porcentaje de las diferencias entre ensambles. Estas correspondieron a las familias numéricamente dominantes, en particular Muscidae y Chironomidae. Dos familias (Chloropidae, Ceratopogonidae), aunque con menor representación numérica total $(<3 \%)$, contribuyeron a establecer las diferencias observadas en la comparación verano medio/verano final. Ninguna de las familias con registro único en las comparaciones entre fases (Tablas 2 a 4) figuró contribuyendo a la separación entre ensambles. Esta situación se debió a la baja representación numérica de estas familias en la vega Tambo-Puquíos (Tabla 1).

Consistente con su condición de prado húmedo, se registraron familias con requerimientos tanto terrestres como acuáticos. La presencia de familias con requerimientos terrestres puede interpretarse como una muestra de la influencia del ambiente terrestre circundante o de los ciclos de sequedadhumedad característicos de estos ambientes. Muscidae, Chironomidae, Syrphidae, Ephydridae, Dolichopodidae y Sphaeroceridae resultaron ser las 
Tabla 9. Comparación entre fases fenológicas de verano de la similitud del ensamble de familias de Diptera registradas en la vega Tambo-Puquíos. Abundancia promedio por fase fenológica de las familias con mayor contribución porcentual a las diferencias observadas. Disimilitud promedio y por familia. En paréntesis, contribución porcentual por familia a las diferencias observadas*

\begin{tabular}{|c|c|c|c|c|c|c|}
\hline \multirow[b]{3}{*}{ Familia/Comparación } & \multirow{2}{*}{\multicolumn{3}{|c|}{ Abundancia promedio }} & \multicolumn{3}{|c|}{ Disimilitud promedio y por familia } \\
\hline & & & & \multirow{2}{*}{$\frac{\mathrm{VI} / \mathrm{VM}}{47,41}$} & \multirow{2}{*}{$\frac{\mathrm{VI} / \mathrm{VF}}{63,73}$} & \multirow{2}{*}{$\frac{\mathrm{VM} / \mathrm{VF}}{50,40}$} \\
\hline & VI & VM & VF & & & \\
\hline Muscidae & 3474,00 & 1384,67 & 199,67 & $16,07(33,90)$ & $40,45(47,78)$ & $18,19(36,10)$ \\
\hline Chironomidae & 3150,00 & 871,33 & 461,33 & $18,32(36,66)$ & $17,46(27,31)$ & $12,16(24,13)$ \\
\hline Ephydridae & 424,67 & 123,00 & 181,67 & $3,14(6,62)$ & $3,79(5,95)$ & $2,42(4,80)$ \\
\hline Dolichopodidae & 281,67 & 401,67 & 1,33 & $2,34(4,94)$ & $3,60(5,65)$ & $5,69(11,29)$ \\
\hline Sphaeroceridae & 400,00 & 38,00 & 4,33 & $2,31(4,87)$ & $2,77(4,36)$ & - \\
\hline Syrphidae & 825,33 & 896,00 & 827,00 & $1,74(3,66)$ & - & $4,46(8,84)$ \\
\hline Chloropidae & 127,67 & 87,33 & 0,33 & - & - & $1,54(3,05)$ \\
\hline Ceratopogonidae & 103,33 & 124,67 & 74,67 & - & - & $1,02(2,02)$ \\
\hline
\end{tabular}

* Prueba SIMPER de una vía para la composición numérica del ensamble sobre los datos logarítmicamente transformados $(\log (\mathrm{x}+1))$.

familias numéricamente dominantes del ensamble. Este grupo de familias tiene valencia ecológica amplia, siendo tolerante a los efectos de las condiciones ambientales extremas o perturbaciones (Keiper et al., 2002). Según estos autores relativamente pocas especies de Muscidae viven en ambientes semiacuáticos. Estas especies poseen larvas que son depredadoras generalistas de invertebrados acuáticos o saprófagos. Algunas especies poseen larvas perforadoras que viven en tallos de juncáceas, elementos florísticos comunes en vegas altoandinas, aunque no en la vega estudiada (Squeo et al., 1994). No obstante, debido al nivel de resolución taxonómica disponible, no es posible informar si Muscidae en el área estudiada está conformada por especies de vega andina o provienen de áreas aledañas, entre ellas aquellas ocupadas por la actividad minera. La presencia de Syrphidae puede deberse al hecho de que las formas inmaduras de diversas especies saprófagas de esta familia ocupan hábitats acuáticos y semi-acuáticos. Representantes de Ephydridae, por su parte, se encuentran frecuentemente y en gran abundancia en ambientes acuáticos y semiacuáticos, particularmente en sus márgenes lodosos, donde se alimentan de organismos pequeños, algas, materia orgánica y excrementos. Estos últimos constituyen elementos relativamente abundantes en la vega estudiada por la entrada de aves acuáticas, guanacos y ganado doméstico. Representantes de Dolochopodidae se encuentran frecuentemente en ambientes terrestres húmedos, donde depredan pequeños insectos. Tanto efídridos como dolicopódidos han sido reportados en lagos salinos del altiplano boliviano (Williams et al., 1995). La presencia de representantes de Sphaeroceridae está asociada a materia orgánica en descomposición (Marshall, 1994). Chironomidae, considerada una familia de especies oportunistas, es común en la mayoría de los ecosistemas acuáticos de montaña (Oertli et al., 2008).

El agua que mantiene los humedales altoandinos del desierto transicional de Chile presenta niveles elevados de $\mathrm{Cu}, \mathrm{Mn}, \mathrm{Fe}, \mathrm{As}, \mathrm{Fl}$, sulfato y elementos traza (Cepeda-Pizarro, 2013). Estas características químicas de los hábitats explotados por insectos andinos pueden representar las fuerzas selectivas que han determinado la composición específica del ensamble de Diptera a encontrar en estos ambientes, como lo han documentado diferentes autores respecto de humedales de otras latitudes (p. e., Márquez-García et al., 2009). No obstante, ya que el estado del conocimiento taxonómico no lo permite, no es posible indicar si las familias registradas en el humedal estudiado en este trabajo están conformadas por especies que son propias de los ecosistemas acuáticos altoandinos. En atención a la estacionalidad del ambiente estudiado, en este trabajo se pretendió evaluar tanto el papel jugado por las familias abundantes (i. e., aquellas con abundancia relativa $\geq 0,1 \%$ ) como aquellas raras (i. e., abundancia relativa $<0,1 \%$ ) (Tabla 1 ), acerca de las características de diversidad y equitabilidad del ensamble de Diptera. Para estos propósitos se usaron índices que entregaran información complementaria respecto de estos grupos. De acuerdo a ello, los 
indicadores de diversidad y equitabilidad $\mathrm{F}$ mostraron que la fase fenológica de verano más favorable a la presencia de Diptera en la vega Tambo-Puquíos corresponde al verano medio, efecto probablemente generalizable a otros prados húmedos altoandinos del desierto transicional de Chile. Basados en estudios vegetacionales, Squeo et al. (2006b) han sugerido la importancia de la longitud de la estación crecimiento. En el área, la precipitación -principalmente nivaltiene una distribución concentrada en los meses de abril a octubre, con máximos entre mayo y agosto. La depleción de la curva mensual de la temperatura, por su parte, coincide con estos meses (Squeo et al., 200b). El examen más detenido del comportamiento de las temperaturas ambientales mínima y máxima del área de estudio, basado en una serie de tiempo de 20 años (Cepeda \& Novoa, 2006), muestra que estas comienzan a aumentar desde un mínimo promedio cercano a $-16{ }^{\circ} \mathrm{C}$ registrado en el período de junio/agosto a un máximo promedio cercano a $20^{\circ} \mathrm{C}$ en enero-febrero, para luego tender a decrecer nuevamente en dirección al invierno. A partir de estos antecedentes climáticos se sospecha que el comportamiento observado del ensamble de familias de Diptera, tanto en términos de su composición como en términos de relaciones numéricas entre sus miembros, estaría aparentemente más relacionado con el patrón térmico del área que con la disponibilidad de microhábitats, evidenciando la importancia de la extensión de la estación de crecimiento. Efectos similares han sido observados en ambientes de montaña de otras latitudes (Füderer et al., 2006).

\section{Conclusiones}

Se registró la presencia de 27 familias de Diptera, con un número estimado de 30 . Seis fueron las familias numéricamente dominantes: Muscidae, Chironomidae, Syrphidae, Ephydridae, Dolichopodidae y Sphaeroceridae. La presencia de familias con especies de hábitos terrestres se interpreta como una muestra de la influencia del ambiente circundante sobre el pastizal húmedo. Las pruebas aplicadas y los índices comunitarios usados apoyan la idea que la estructura del ensamble de Diptera de esta vega altoandina varía rápidamente durante el curso de la estación de crecimiento, variación aparentemente debida más al patrón térmico de la montaña que a la disponibilidad de microhábitats favorables a los ciclos biológicos de las especies constituyentes del ensamble.

\section{Agradecimientos}

Esta publicación fue financiada por la Dirección de Investigación de la Universidad de La Serena. Hernán Vásquez C. colaboró con los trabajos de terreno y el procesamiento del material capturado.

\section{Literatura Citada}

Anderson, M.J.

2005. PERMANOVA Permutational multivariate analysis of variance. A computer program. Departmento of Statitics. University of Auckland. New Zeland.

Batzer, D.; Wissinger, S.A.

1996. Ecology of insect communities in nontidal wetlands. Annual Review of Entomology, 41: 75-100.

Cepeda P., J.; Novoa, J.

2006. La cordillera altoandina del Valle del Elqui. En: Geoecología de los Andes desérticos. La Alta Montaña del Valle del Elqui. Cepeda P., J. (ed.). Ediciones Universidad de La Serena. La Serena, Chile, pp. 41-63.

Cepeda-Pizarro, J.; Pola P., M.

2013. Relaciones de abundancia de hexápodos terrestres en vegas altoandinas del desierto transicional de Chile. Idesia, 31:31-39.

Cepeda-Pizarro, $\mathrm{J}$.

2013. Variabilidad temporal de algunos parámetros físicos e hidroquímicos de un prado húmedo altoandino del nortecentro de Chile. Idesia, 31: 89-97.

Contreras, J.

2002. Norte de Chile: conservación de humedales altoandinos para un desarrollo productivo sustentable. Revista Ambiente y Desarrollo, Chile, Vol XVIII: 125-131.
Füderer, L.; Ettinger, R.; Boggero, A.; Thaler, B.; Thies, H. 2006. Macroinvertebrate diversity in Alpine lakes: effects of altitude amd catchment properties. Hydrobiologia, 562: 123-144.

Henderson, P.; Seaby, R.

2008. A Practical Handbook for Multivariate Methods. Pisces Conservation IRC House, The Square, Pennington, Lymington, Hants, United Kingdom. 224 p.

Hinden, H.; Oertli, B.; Menetrey, N.; Sager, L.; Lachavanne, J.B. 2005. Alpine pond biodiversity: what are the related environmental variables. Aquatic Conservation. Marine and Freshwater Ecosystems, 15: 613-624.

Keiper, J.B.; Walton, W.E.; Foote, B.A.

2002. Biology and ecology of higher Diptera from freshwater wetlands. Annual Review of Entomology, 47: 207-232.

Márquez-García, M.; Vila, I.; Hinojosa, L.F.; Méndez, M.A.;

Carvajal, J.L.; Sabando, M.C.

2009. Distribution and seasonal fluctuations in the aquatic biodiversity of the southern Altiplano. Limnologica, 39: 314-318.

Marshall, S.A.

1994. Peatland Sphaeroceridae (Diptera) of Canada. Memoirs of the Entomological Society of Canada, 126: 173-179. 
McAlpine, J.F.; Peterson, B.V.; Shewell, G.E.; Teskey, H.J.; Vockeroth, J.R.; Wood, D.M. (eds.).

1981. Manual of Neartic Diptera. Monograph 27 (Volume 1). Research Branch, Agriculture Canada. Canada.

McAlpine, J.F.; Peterson, B.V.; Shewell, G.E.; Teskey, H.J.; Vockeroth, J.R.; Wood, D.M. (eds.).

1987. Manual of Neartic Diptera. Monograph 28 (Volume 2). Research Branch, Agriculture Canada. Canada.

Oertli, B.; Indermuehle, N.; Angelibert, S.; Hinden, S.; Stoll, A. 2008. Macroinvertebrate assemblages in 25 high alpine ponds of the Swiss National Park (Cirque of Macun) in relation to environmental variables. Hydrobiologia, 597: 29-41.

Rosenberg, D.M.

1992. Freshwater biomonitoring and Chironomidae. Netherland Journal of Aquatic Ecology, 26: 101-122.

Seaby, R.M.; Henderson, P.A. 2006. Species Diversity and Richness Version 4. Pisces Conservation Ltd. Lymington, England. 119 p.

Squeo, F.A.; Warner, B.G.; Aravena, R.; Espinoza, D. 2006a. Bofedales: high altitude peatlands of the Central Andes. Revista Chilena de Historia Natural, 79: 245-255.
Squeo, F.A.; Ibacache, E.; Warner, B.; Espinoza, D.; Aravena, R.; Gutiérrez, J.

2006b. Productividad y diversidad florística de la vega Tambo, cordillera de Doña Ana. En: Geoecología de los Andes desérticos. La Alta Montaña del Valle del Elqui. Cepeda P., J. (ed.). Ediciones Universidad de La Serena. La Serena, Chile, pp. 325-351.

Squeo, F.A.; Osorio, R.; Arancio, G.

1994. Flora de los Andes de Coquimbo. Cordillera de Doña Ana. Ediciones Universidad de La Serena, La Serena, Chile. $168 \mathrm{pp}$.

Steel, R.G.D.; Torrie, J.H.

1989. Bioestadística. Principios y Procedimientos. McGraw Hill, México D.F., México. 622 pp.

Timms, L.L.; Bowden, J.J.; Summerville, K.S.; Buddle, C.M. 2014. Does species-level resolution matter? Taxonomic sufficiency in terrestrial arthropod biodiversity studies. Insect Conservation and Diversity, 6: 453-462.

Williams, W.D.; Carrick, T.R.; Bayly, I.A.E.; Green, J.; Herbst, D.B. 1995. Invertebrates in salt lakes of the Bolivian Altiplano. International Journal of Salt Lake Research, 4: 65-77. 